\title{
A Unified Emergency Care System from First Aid to Definitive Care
}

\author{
Lt Col D Morgan-Jones \\ MBE, MRCGP, DMCC, RAMC \\ Chief Instructor \\ Defence Medical Services Training Centre, Keogh Barracks, Ash Vale, Aldershot, Hants GU12 5RQ \\ e-mail:d.morgan-jones@virgin.net
}

\author{
Lt Col TJ Hodgetts \\ FRCP, FFAEM, DipIMC RCSEd, DipMedEd, RAMC \\ Consultant and Specialty Adviser in Accident \& Emergency, \\ MDHU Frimley Park Hospital, Portsmouth Road, Frimley, Surrey GU16 $5 U J$
}

\author{
Professor of Emergency Medicine and Trauma \\ University of Surrey \\ e-mail: tim.hodgetts@virgin.net
}

SUMMARY: The Unified Emergency Care System (UECS) provides an integrated system of medical support from point of injury to the time a casualty is handed over to specialist care within hospital. It enables personnel at all skill levels to deliver life-saving support to casualties with a broad range of acute injuries and illness. The UECS facilitates standardised training with each level building upon the previous, yet it retains an inherent flexibility to adapt to specific operational and service requirements.

\section{Introduction}

Experience from previous conflicts has demonstrated how essential the provision of effective pre-hospital care can be in minimising deaths on the battlefield (1-3). In most situations it will be the combination of self-help, buddy aid and the actions of regimental medics at platoon and company level which will have the greatest impact. If the battlefield becomes less dense, as is presently postulated, then the role these individuals will play keeping casualties alive will become even more important. However, whilst military planners continue to focus on the high intensity component of the operational spectrum, the reality of present operations and the need to provide a level of care comparable to that provided by the NHS, means that we must examine how we train and equip each component (see Text Box 1) of the pre-hospital care system. This issue is not new $(4,5)$, but perhaps the reason why no progress has been made is the lack of a fully integrated, clinically orientated acute care doctrine, upon which training and the provision of equipment can be based.

\section{- Basic Aid A level of care provided by non-medical personnel "First Aid". \\ -Intermediate Any individual "Medic" taught to Aid provide augmented first aid. \\ - Advanced Aid An individual "paramedic" who has been taught to Institute of Health and Care Development (IHCD) standards and who also has the appropriate experience.* \\ * This status does not presently exist within the Defence Medical Services - PHIC MO

$$
\begin{aligned}
& \text { A doctor who is either a specialist } \\
& \text { primary care physician or whose job } \\
& \text { involves working temporarily within the } \\
& \text { pre-hospital environment. }
\end{aligned}
$$

NOTE: Whilst a patient could pass through each of these elements, it is more likely that some stages would be omitted.

Text Box 1. Elements of the Pre-Hospital Care Chain.

\section{Requirements}

There is an increasing requirement to provide evidence-based best practice across the spectrum of medical care. Evidencebased medicine combines the best available clinical research with the best clinical experience. To rely on research alone will result in excessively pragmatic protocols that will be inflexible for every clinical situation - and to rely on clinical experience alone will result in practice excessively influenced by personal anecdote(6). In an ideal system, therefore, the following will be required for the most effective military pre-hospital care:

1. An integrated and seamless casualty management system that starts from the point of injury and ends (for the pre-hospital element) with the patient receiving the appropriate specialist hospital-based care.

2. A system that will enable the individual to manage casualties suffering from a wide spectrum of illness and injury (that is, not focused entirely on trauma).

3. A stepwise evidence-based training programme for progressive levels of pre-hospital care providers that will meet present and potential threats. Such a programme must be fundamentally simple to teach. It will accept that skill fade will occur, but will accommodate top up training and just-in-time (JIT) training with 'tailor made' modules for specific operations.

\section{The status quo}

The Defence Medical Services teach a multiplicity of first aid and para-medical courses. Within the Defence Medical Services Training Centre this includes 8 different first aid courses and 18 variations of para-medical training programmes. Whilst the management of trauma is taught it is by no means evidencebased, and only minor elements of the hazard spectra (trauma, medical/toxicology and environmental emergencies) are covered. For doctors entering primary care, the acute management of trauma, medical emergencies (cardiac incidents only) and chemical casualties are taught-but within separate and unconnected courses. This approach is further confused by a hospital-based approach being imposed onto the pre-hospital environment. An added disadvantage to this fragmented instructional process is the resultant diversity of medical equipment recommendations. 
Present casualty management systems tend to be seduced by the pure therapeutic element of a patient's care. However, this aspect is only part of the patient's overall immediate management, particularly when dealing with the peculiarities of military practice. The other factors that need to be taken into account are illustrated in Figure 1. Safety is a significant issue when delivering acute care during peacetime practice and for operations other than war (OOTW). A dead medic is of use to no one. During battle risks have to be taken, but they can be minimised through effective procedures and training. The requirement for triage is well recognised (7-10). Extrication of casualties, particularly from armoured vehicles, will be a major problem. It is an area where both doctrine and skills need to be developed. Treatment will be discussed later in this article. Following treatment for acute life-threatening injuries casualties may require packaging (for example, with splints, dressings, fluids and medication) prior to evacuation. Due to the vagaries of the battlefield a casualty may have to be held for some hours - and therefore it is essential that individuals throughout the pre-hospital care chain have the appropriate level of nursing skills for looking after acutely ill patients.

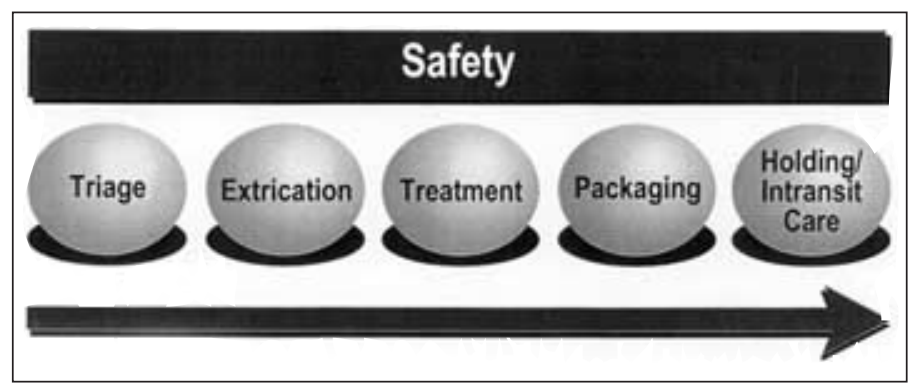

Fig 1. Casualty Management Factors

\section{The Way Forward}

The UECS provides a single, systematic, "all hazard" approach to the management of acute emergencies in medicine from point of injury to definitive clinical support. This is irrespective of whether the insult is traumatic, medical, toxicological or environmental in nature. The 'ABC' (Airway, Breathing and Circulation) approach is used to provide the basis for acute care irrespective of skill level. The key elements for each level of skill have been identified. The management templates take into account best clinical experience and, where it exists, the best available clinical evidence.

The icon-based system facilitates a rapid and concomitant consideration of clinical problems across the spectrum of acute emergencies. An additional dove (for peace) or cross swords (for operations) alongside an icon will clearly identify where in the operational spectrum it is appropriate to be applied. For example, the management of chemical casualties would normally be undertaken only during conflict-so the cross swords appear; and a spinal board has no place in an infantry section-so the dove appears.

Using the presently accepted stages of treatment (Text Box 1) the skills and procedures for the primary survey and immediate management required for each link of the treatment chain can be clearly articulated using the UECS approach:

- Basic Aid (Fig 2). Brings together the basic management of trauma, cardiopulmonary resuscitation (CPR, Basic Life Support) and the initial management of chemical casualties (Fig 3).

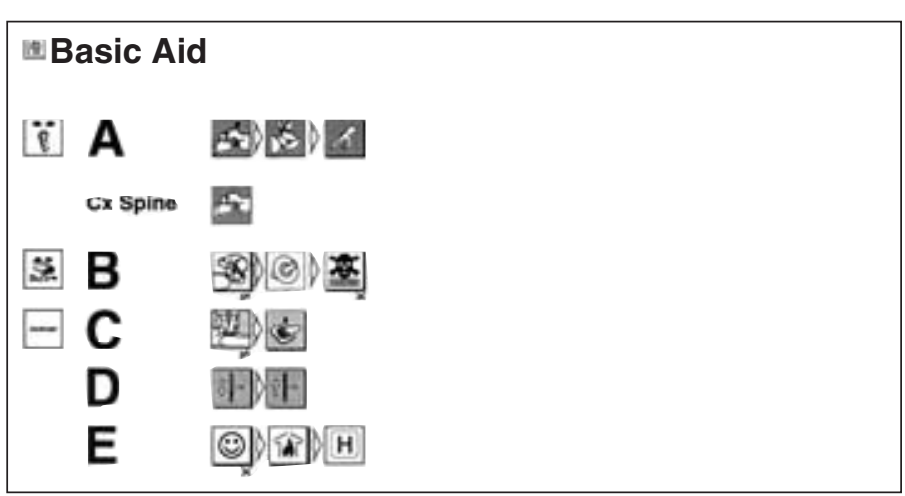

Fig 2. Primary Survey - Basic Aid

- Intermediate Aid(Fig 4). At this level there is a continuing pressure to add complex skills (for example endotracheal intubation, and IV cannulation) to a medic's armamentarium. This has been resisted. A lack of practical experience and judgement may otherwise draw the medic to perform a complex skill badly (compounded by the difficult working environment), at the expense of performing simple skills well (for example, poor control of external haemorrhage at the expense of IV cannulation).

\section{Intermediate Aid

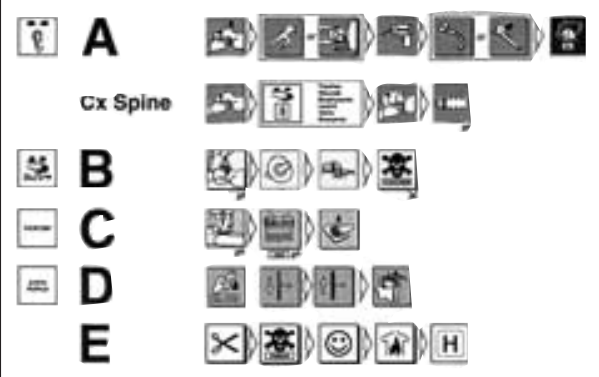

Fig 4. Primary Survey - Intermediate Aid.

- Advanced Aid (Fig 5). The paramedic tier builds on the skills of the medic. In its current form it acts as a framework for discussion with the IHCD with regard to the capabilities that will be expected of future military paramedics.

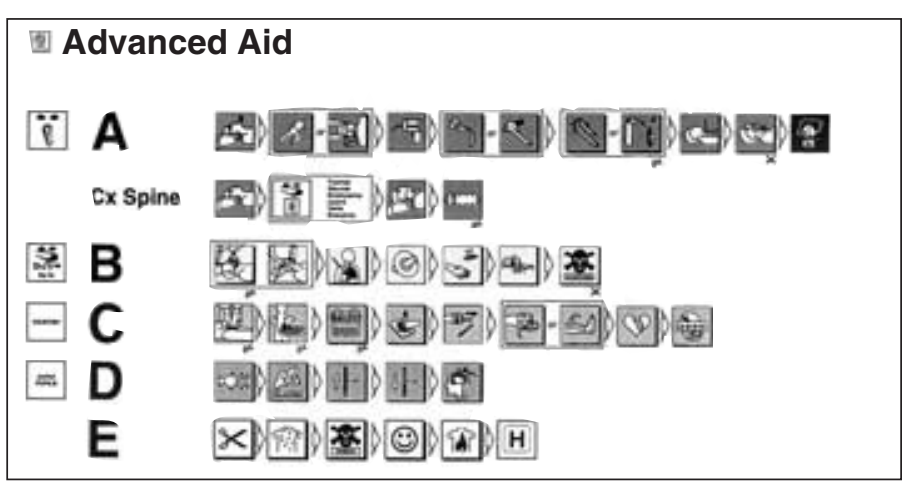

Fig 5. Primary Survey - Advanced Aid

- Pre-Hospital Medical Officer (Fig 6). The principal differences that determine the management by the prehospital medical officer compared to the emergency physician are the limited monitoring systems available, the limited complexity of the treatment protocols, and the absence of definitive intensive care or surgical support. For example, it can be reasonably expected in the pre-hospital environment to treat an adult with status epilepticus using simple airway adjuncts and oropharyngeal suction, oxygen, 


\section{Icon Glossary}

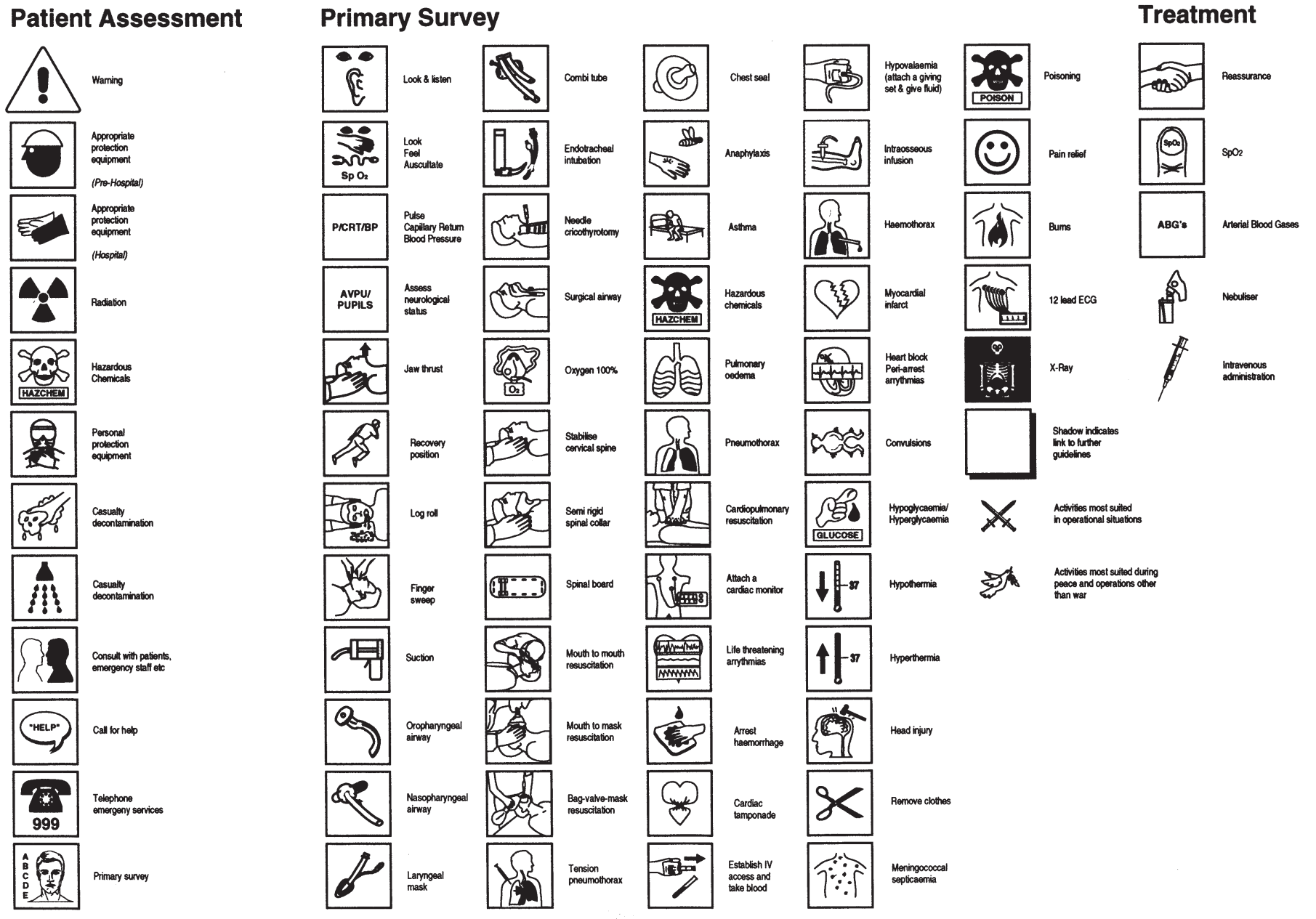

Fig 3. The Icon Glossary

and repeated doses of intravenous benzodiazepine. In the A\&E department these measures are a first option, but there is the opportunity for further therapeutic intervention (for example, intravenous phenytoin) and ultimately for rapid sequence induction, endotracheal intubation, and control of convulsions with a barbiturate anaesthetic agent.

\section{Pre-Hospital Intermediate Care MO}

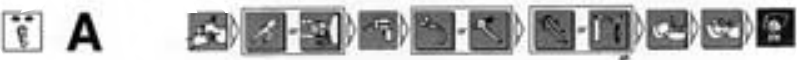

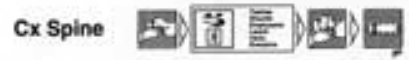

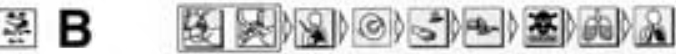

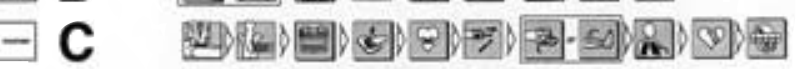

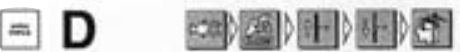 \\ E $x$ 国国}

Fig 6. Primary Survey - Pre-Hospital Medical Officer

- The Emergency Physician (Fig 7) provides the full depth and breadth of emergency treatment currently available in today's civilian medical practice. This capability would be expected to be available across the conflict spectrum.

\section{圆 Emergency Physician}

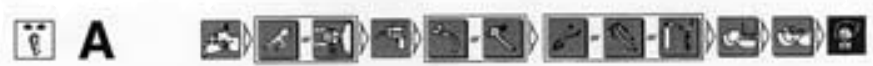

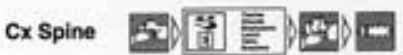

B 3 B

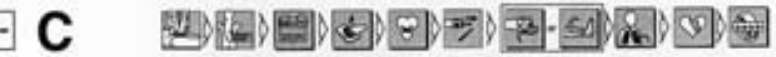

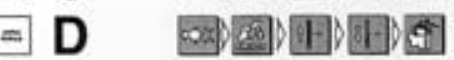

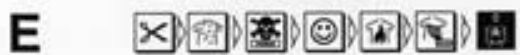

Fig 7. Primary Survey - Emergency Physician

\section{Conclusion}

The UECS provides an integrated, seamless, evidencebased approach to emergency care across the conflict spectrum. It is the platform for the development of a system of medical support from point of injury to the time a casualty is handed over to specialist care within hospital. It enables personnel at all skill levels to provide life-saving support to casualties suffering from a wide range of acute injuries and illness. This approach facilitates a standardised training approach with each level building upon the previous, but retains an inherent flexibility allowing it to adapt to specific operational and service requirements. 
By defining the capabilities at each level the UECS provides the template for the rational development of equipment scales. It is a radical and new concept that challenges the current established fragmented teaching of medical emergencies. Academic support leading to external accreditation is required.

\section{REFERENCES}

1. Bellamy RF. The Causes of Death in Conventional Land Warfare; Implications for Combat Casualty Care Research. Milit Med 1984; 149: 55-62.

2. Bellamy RF. Death on the Battlefield and the Role of First Aid. Milit Med 1987; 152: 634-5.

3. Gofrit N. Leibivici D, Shapira SC, Shemer J, Stein M, Michaelson M. The Trimodal Death Distribution of Trauma Victims: Military Experience from the Lebanon War. Milit Med 1997; 162: 24-26.
4. Bricknell MCM. First Line Medical Support - The Need for an Integrated Package. BAR Aug 1991; 98: 53-59.

5. LYNCH P. First Line Medical Support: Time It Was Sorted Out. BAR Feb 1997; 114: 55-58.

6. Sackett D, Richardson W, Rosenberg W, Haynes R. Evidence-based medicine: how to practice and teach EBM. London: Churchill Livingstone, 1997.

7. Hodgetts T. Triage. Pre-hospital Immediate Care 1997; 1: 110-111.

8. Hodgetts T, Morgan-Jones D. Triage and initial documentation of nuclear/radiation casualties. Prehospital Immediate Care 1998; 2: 165-168.

9. Advanced Life Support Group. Major Incident Medical Management and Support: the practical approach. London: BMJ Publishing, 1995.

10. Hodgetts T, Hall J, Maconochie I, Smart C. Paediatric triage tape. Pre-hospital Immediate Care 1998; 2: 155-159. 
Page 136

Full Page Mono Advert

Territorial Army

Bromide Supplied 